Bioscientia Medicina: Journal of Biomedicine \&

Translational Research

Journal Homepage: www.bioscmed.com

\title{
Understanding Anorexia and Eating Capability of Older Adults in Attempt to Prevent Malnutrition
}

\section{Afifah Amatullah ${ }^{*}$, Roza Mulyana ${ }^{2}$}

${ }^{1}$ Internal Medicine Department, Faculty of Medicine, Andalas University, Padang, Indonesia

2 Geriatric Division of Internal Medicine Department, Faculty of Medicine, Andalas University/Dr M Djamil General Hospital, Padang, Indonesia

\author{
A R T I C L E I N F O \\ Keywords: \\ Aging \\ Eating capability \\ Malnutrition \\ Older adults \\ *Corresponding author: \\ Afifah Amatullah \\ E-mail address: \\ afifahamatullah@gmail.com
}

\begin{abstract}
A B S T R A C T
Because of the rise of life expectancy, the older adults are now a big part of world's population. In United States, prevalence of adults aged more than 65 years old increased from 36.6 million in 2005 to 47.8 million in 2015. In Indonesia, percentage of older adults increased two-fold from $1971-2019$ as much as 9,6\% or more than 25 million people. Along with this aging population, eating problems and malnutrition are getting more frequent. Aging causes several changes and degradation of body function which physiologically lead to anorexia and eating difficulty in older adults. Moreover, the other age-related factors such as social factor, economic factor, psychological factor, diseases and polypharmacy also have significant impact on food intake in older adults. Prevalence of inadequate energy intake and malnutrition in older adults are increasing, which lead them to become susceptible to acute and chronic diseases or worsening of their disease, increase hospitalization, and increase mortality rate. Even so, the signs of malnutrition of older adults are often late to be recognized, whereas the recovery of malnutrition in older adults is much more complicating than in younger adults. This review discusses about problems encountered by older adults that decrease their intake and cause malnutrition, to help clinicians recognize the cause of malnutrition in older adults earlier.
\end{abstract}

All authors have reviewed and approved the final version of the manuscript.

https://doi.org/10.37275/bsm.v6i1.439

\section{Introduction}

Because of the rise of life expectancy, older adults have become a big portion of world's population. In United States, prevalence of adults aged more than 65 years old increased from 36.6 million in 2005 to 47.8 million in 2015. ${ }^{1}$ Prevalence of older adults in Indonesia increased two fold from 1971 until 2019. In 2019, there are $9.6 \%$ or more than 25 million older adults in Indonesia. ${ }^{2}$ As the population getting older, feeding problems and malnutrition are becoming more prevalent and being discussed more often.

Nutrition has a critical role in aging process and health status, it helps maintaining good health and reduces the risk of chronic illness. Malnutrition is a state of poor nutrition, comprises undernutrition and overnutrition of macronutrients and/or micronutrients. Malnutrition in older adults increases the risk of suffering poor health or chronic illness, including sarcopenia and cardiovascular diseases. ${ }^{3}$

Prevalence data showed that macronutrients (protein-energy) and micronutrients (vitamin and mineral) deficiency are common in older adults. Approximately $40-50 \%$ older adults are having moderate to high risk of nutrition problems, and up to $40 \%$ are deficient of three or more nutrients. ${ }^{4}$ Malnutrition is reported in $5-10 \%$ among communitydwelling older adults, 30 - 61\% among hospitalized older adults, and $12-85 \%$ among older adults in longterm care facilities.

As someone ages, energy intake is decreased significantly as result of decreased lean body mass 
(LBM) and physical activities. In many nutrition surveys, most of community-dwelling older adults have a decreased calorie intake below daily requirement. The National Health and Nutrition Examination Survey (NHANES) III reported that the average daily energy intake in individuals aged more than 70 years old is approximately $1800 \mathrm{kcal} /$ day for men, $1400 \mathrm{kcal} /$ day for women, and less than $1000 \mathrm{kcal} /$ day in more than $10 \%$ older adults. With limited energy intake, the required calorie in inactive older adults could be meet, but the required vitamin and mineral would still be lacking. ${ }^{4}$

Nutrition status in older adults is very susceptible to any stressor. When their intake is decreased, they would be in risk of malnutrition. Energy-protein deficiency is the most common form of malnutrition and often unrecognized in most cases. Moreover, the consequences of malnutrition in older adults are obscure and noxious compared to younger adults, and the recovery is complicated. 5 Poor nutritional status is associated with impaired immunity, impaired wound healing, decreased functional status, increased hospitalization and mortality. Non-nutrition factors cannot be eliminated, nevertheless poor nutrition is known as independent factor of increased morbidity and mortality. Numerous evidences showed that measurable benefits are obtained with better nutrition status. 6

Multiple factors can lead to decreased intake in older adults. Aging itself is characterized with gradual decrease of body function that will interfere their eating process. Psychological, economic, social, and illness have a significant impact on nutrition intake in older adults. Adequate intake is critical to prevent malnutrition in older adults, therefore any eating difficulties should be identified early. This review discusses problems that will cause inadequate nutrition intake in older adults as additional knowledge for clinicians to help them identify and provide education to the patients and their families.

\section{Nutrition in older adults}

Aging process leads to several changes within the body which needed to be carefully considered even with optimal lifestyle. Naturally, aging process induces nutrition deficiency which should be monitored even in the healthy patients. Oftentimes, vitamin requirement increases at the same time as decreased appetite and reduced physical capacity, leaving the older adults in vulnerable state to deficit nutrient reserves. Vitamin B, $\mathrm{HCl}$, vitamin $\mathrm{D}$, iron, zinc, selenium and magnesium deficiencies could cause significant impact to health status of older adults. ${ }^{7}$

The goals of improvement of nutrition status in older adults are to maintain or promote general health and quality of life, and to prevent or treat health problems that related to age and nutrition. Optimal nutrition status is not only beneficial to overall health, but also to delay aging process, affect physiological system and function, body composition, along with onset and management of chronic conditions. 8

Aging process is known with its direct role in changes of nutritional requirements. With aging, some nutrients are needed more, less or stay the same. Changes in social and economic status lead to reduced access to food, reduced food choices, nutrient deficiency, and poor nutritional status. These circumstances result to increased risk of illness, poor health, limited mobility and dependency, as shown in figure 1.8 


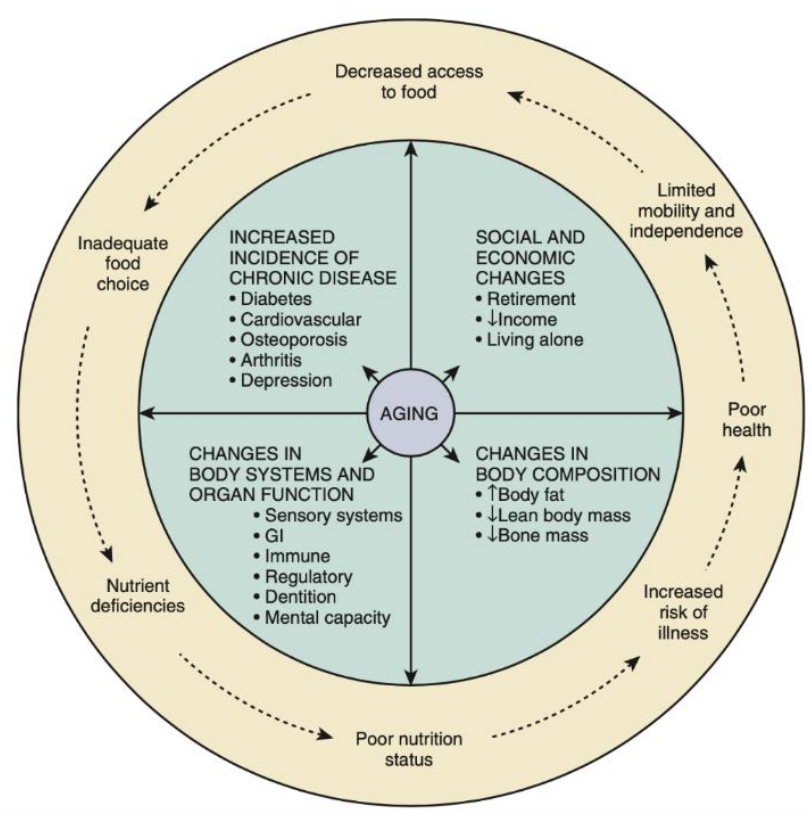

Figure 1. The role of age-related changes to poor nutritional status and increased risk of illness in aging cycle. 8

\section{Age-related physiological changes \\ Physiological changes of gastrointestinal system}

Aging causes impairment of gustatory and olfactory sensations, thus causing altered cephalic phase of digestion. Sensory outputs including sight and smell before eating are mediators in cephalic phase for regulating appetite and food ingestion. 1 Shape, flavor, taste, and texture of the foods are all take effect in one's desire of eating and give stimulation to continue eating or not. The ability to smell and taste foods are crucial to enjoy the foods, and are important regulators in nutrition intake. Disruption of those abilities as shown in table 1 will result in altered eating habit and the urge to maintain adequate intake. 9

Table 1. Processes in oropharynx of older adults 10

\begin{tabular}{|l|l|l|}
\hline Process & Healthy older adults & $\begin{array}{l}\text { Older adults with complex } \\
\text { clinical condition }\end{array}$ \\
\hline Taste & Unaffected & Diminished \\
\hline Smell & Diminished & Diminished \\
\hline Food enjoyment & Unaffected & Diminished \\
\hline Salivary output & Unaffected & Diminished \\
\hline Chewing efficiency & Slightly diminished & Diminished \\
\hline Swallowing & Slightly diminished & Diminished \\
\hline
\end{tabular}

The older adults have higher threshold to be able to recognize sweetness, bitter, salty or sour compared to younger adults. Difficulty in distinguishing tastes is caused by age-related changes in olfactory epithelial, neural and receptor pathways. Flavor and taste of food are needed in giving enjoyment while eating, and also help to recognize certain foods. Older adults tend to eat more if the food was given flavor enhancement, but they used to choose similar diet instead of improving the taste of their food. Older adults often let go the food enjoyment and make them in increased risk of malnutrition. ${ }^{11}$

Oropharynx, upper esophageal motility, colonic function, gastrointestinal immunity, and drug metabolism are significantly affected by the aging process. Several changes that related to age in gastrointestinal system are constipation, swallowing disorder, esophageal reflux, dysmotility syndrome, and neoplasia of GI tract. Effects of aging on gastrointestinal tract are shown in figure 2 below. 9 


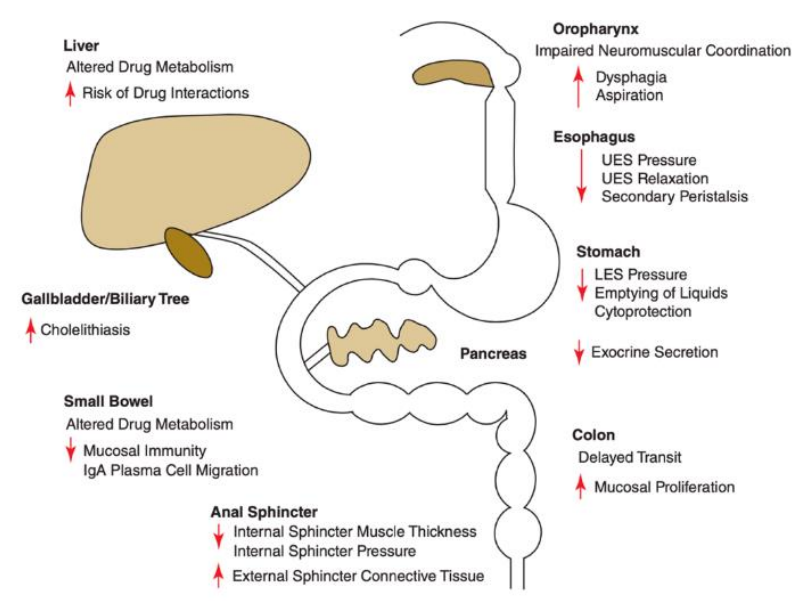

Figure 2. Physiological effects of aging on gastrointestinal tract 9

Swallowing process requires complex coordination of motoric, sensory, and psychological activities, both voluntary and involuntary activities. With aging, several changes occur in swallowing function known as presbyphagia. Presbyphagia refers to natural changes in swallowing process of a healthy older adults, while dysphagia or swallowing disorder is a pathologic condition which commonly suffered by older adults. In oral phase of swallowing, normal aging is associated with loss of dentition, poor oral hygiene, decreased sensation of taste and smell, decreased ability of determining texture, hyposalivations along with xerostomia, and reduced strength of tongue and mastication muscles. 12

Pharyngeal phase of swallowing is getting slower as someone ages, which is characterized by pharyngeal muscles atrophy and increased size of pharyngeal lumen.11 The function of oropharynx muscle and relaxation of upper esophageal sphincter also slowed down with aging thus delay food transfer to pharynx. Inability to transfer food to esophagus can cause food to enter the area above vocal cords and allow aspiration into the trachea. It is notable that frequently, dysphagia symptom in older adults is caused by more serious etiology such as malignancy. Older adults are also at risk to various complications of inadequate chronic esophageal disease treatment, such as aspiration, malnutrition and Barrett's metaplasia. 10

Older adults tend to have a longer duration of gastroesophageal reflux as a result of decreased peristaltic movements and impaired contraction of lower esophageal sphincter. Changes in myenteric plexus related to aging also lead to impairment of gastric emptying, which cause distention and early satiety in older adults. ${ }^{1}$ Intestinal motility also affects the balance of hunger and satiety at colon-rectum level. Stasis in the colon slows gastric emptying via colongastric reflex, and indirectly prolongs the sense of satiety. ${ }^{11}$

\section{Social, economic and cultural factors}

From social perspective, maintaining healthy and balance eating pattern is affected by several factors. Those factors are physical factors including food availability, economic factors including affordability, sociocultural factors including norms and values, political factors including rules and law. Community-dwelling older adults are at higher risk to malnutrition because they are having relative difficulty to obtain food, difficulty in transportation to grocery store, difficulty to pay because of limited income or high medical expenses. 1

Eating habit, culture and faith also affect food choices. Low education and inappropriate understanding about diet restriction lead to inappropriate and fewer food choices. Older adults who live alone, particularly men, are 
having higher risk to malnutrition. This suggests that social isolation is an important risk factor for malnutrition. 1

A good social relation will positively affect appetite and nutrition intake. Friendship, marital status, and togetherness help counter negative effects from financial stress to food intake. For older adults who have lost their partner, mealtime could be a sad moment for reminding them of good memories. 13

Eating is not only a biological action but also involves social and cultural values. Eating patterns such as when, where and how someone eats are decision made based on individual choices dan habits during life. In nursing home, food choices generally are very limited, so older adults often refuse to eat because they don't like the food or it doesn't match their eating habits. Sometimes older adults also refuse to eat in order to gain attention from the officers. 13

\section{Disease and polypharmacy}

Anorexia in older adults can occur as a result from systemic diseases such as chronic heart failure, chronic kidney disease, chronic obstructive pulmonary disease, rheumatologic diseases, cancer, dan acute illness. In those circumstances, systemic inflammation occurs and the body is in hypercatabolic state, which result in cachexia. Pro inflammatory cytokines (IL-6, IL-1, TNF- $\alpha$ ) stimulate serotonergic neuron and melanocortin system in hypothalamus which decrease the appetite. Moreover, there is also inhibition of heavy chain myosin transcription which induces proteolysis through ubiquitinproteasome mechanism and also causes cachexia. ${ }^{1}$

Stress and chronic inflammation activate adrenocortical system and catecholamine which increase basal energy expenditure (BEE) and reactive oxygen species (ROS) then cause protein degradation. Cachexia is a serious wasting disorder, it is not a consequence of starving or age-related muscle loss and unresponsive to high calorie intake. ${ }^{1}$

Dysphagia is a pathologic condition that commonly occurs in older adults, and is an independent factor of malnutrition. The most common prevalence of dysphagia is in older adults with neurological and neuromuscular disorders. Dysphagia is most frequently caused by acute stroke, approximately $51-64 \%$ patients with stroke were having dysphagia. Other neurological disorders which often cause dysphagia are dementia and Parkinson's disease. Up to $93 \%$ patients with dementia were having dysphagia. In patients with Parkinson's disease, there were 20 - 39\% dysphagia from subjective assessment and $82 \%$ from objective assessment. 14

Parkinson's disease, stroke and rheumatoid arthritis are associated with eating difficulty. Symptoms of Parkinson's disease such as rigidity, tremor, slow body motion, swallowing difficulty, and decreased intestinal motility will affect nutrition intake. Those symptoms also use up energy in patients with Parkinson's disease. Rheumatoid arthritis causes joint deformity that makes it difficult for patients to lift pots and pans or to hold knife and fork. Older adults with those diseases need assistance from other people to get sufficient intake. 15

As many of $80 \%$ of stroke patients were having eating difficulty related to less portion of food, difficulty to manipulate food on the plate, transferring food into the mouth, sitting position, eating pace, manipulating food in the mouth, swallowing difficulty, opening and/or closing the mouth, and vigilance. Most of the stroke patients need assistance in eating due to swallowing disorder, pneumonia, forget to eat and decreased appetite. 15 
Inappropriate prescribing is also associated with several side effects such as impaired taste, dry mouth, constipation and nausea, thus decrease food intake. Polypharmacy is an important risk factor of malnutrition in older adults. Several medications have impact on appetite and taste or cause gastrointestinal symptoms like nausea, vomiting and diarrhea (Table 2). ${ }^{1}$

Table 2. Medications that affect taste and appetite ${ }^{1}$

\begin{tabular}{|l|l|}
\hline Medication Class & Side Effects \\
\hline Antibiotics (macrolide, metronidazole, fluoroquinolone) & Dysgeusia \\
\hline Anti-epileptics (carbamazepine, phenytoin) & Dysgeusia, nausea, vomiting \\
\hline Mood stabilizer (lithium) & Dysgeusia \\
\hline Antihypertensive (captopril, ramipril) & Dysgeusia \\
\hline Anti-neoplastic drugs (5-fluorouracil, cisplatin) & Dysgeusia, nausea, vomiting \\
\hline Anti-Parkinsonian drugs (levodopa, amantadine) & Dysgeusia, nausea, vomiting \\
\hline $\begin{array}{l}\text { Anticholinergics (antispasmodics, antimuscarinics, tricyclic anti- } \\
\text { depressants) }\end{array}$ & Dysgeusia, dysphagia \\
\hline Statins (atorvastatin, simvastatin) & Dysgeusia \\
\hline Muscle relaxants (baclofen) & Dysgeusia \\
\hline Digoxin, hormone replacement therapy dan phenytoin & Nausea, vomiting \\
\hline Cholinesterase inhibitors (donepezil, rivastigmine) & Nausea, vomiting \\
\hline
\end{tabular}

\section{Psychological and cognitive disorders}

One of serious mental disorders that often overlooked in older adults is eating disorder, which occurs more commonly in adolescences and young adults. Research showed that desire to be lean and dissatisfaction of body shape can occur in older adults, and have strong association with the fear of aging. Eating disorder in this population is divided into two groups based on its onset, young onset disorder that lingers into old age and late onset disorder that occurs first in older age. Eating disorder that occurs after age 40 years is considered late onset. ${ }^{1}$

Eating disorders in older adults are associated with increased morbidity and mortality. A study found that $21 \%$ of mortality rates are associated with eating disorders in older adults with average age 68,6 years old. Eating disorders should be considered in older adults who experience unexplained loss of body weight. These disorders consist of anorexia nervosa, bulimia nervosa, binge eating disorder, and other eating disorders. ${ }^{1}$
Depression and dementia are major causes of weight loss in older adults which involve $10-20 \%$ weight loss in community-dwelling older adults and $58 \%$ older adults in nursing home. Depression in older adults could appear as primary diagnosis or complication of another underlying disease, and often underdiagnosed. Depression should be considered early in diagnosing weight loss so that the appropriate therapy and adequate intake can be given immediately. 13

Patients with dementia often have malnutrition, loss of body weight and dehydration. Several factors can be the cause of those circumstances, such as anorexia, inadequate oral intake because patient didn't remember to eat, depression, feeding apraxia, or rarely increased energy requirement because of hyperactivity. ${ }^{14}$ Patients may experience agnosia, a difficulty to interpret sensations related to sight, taste, smell, or touch. They may refuse to eat because they cannot recognize the objects as food. 13 


\section{Eating capability in older adults}

Aging causes debility in one's physical, psychological and mental ability. These changes also manifest significantly in eating capability of older adults. Eating difficulty in older adults will reduce their food intake, increase the risk of malnutrition and have big impact on quality of life. 16

Westergern et al. in 2002 studied eating difficulty in older adults who stayed at hospital by observing their eating habits. From this study, difficulties were found when the patients were sitting, manipulating food on the plate, transferring food into the mouth, opening and closing their mouth, and swallowing. From 520 samples in total, $82 \%$ were having eating difficulty, 36\% needed assistance, and 46\% were having risk of malnutrition. They also found strong correlation between eating difficulty and poor energy intake and malnutrition. ${ }^{17}$

During the eating process, several actions should be done in a coordinated manner. Before food ingestion, those actions include manipulating food on the plate with hands or cutlery, lifting food into the mouth, lowering the jaw in order to open the mouth, and others. After food ingestion, a sequence of actions including closing the mouth, biting, masticating, transferring, mixing, saliva secretion, sensory detection, forming bolus and others inside the mouth. To properly do those actions, good abilities in physical, psychological and mental are required. Laguna et al. described those actions as eating capability as shown in figure 3.16

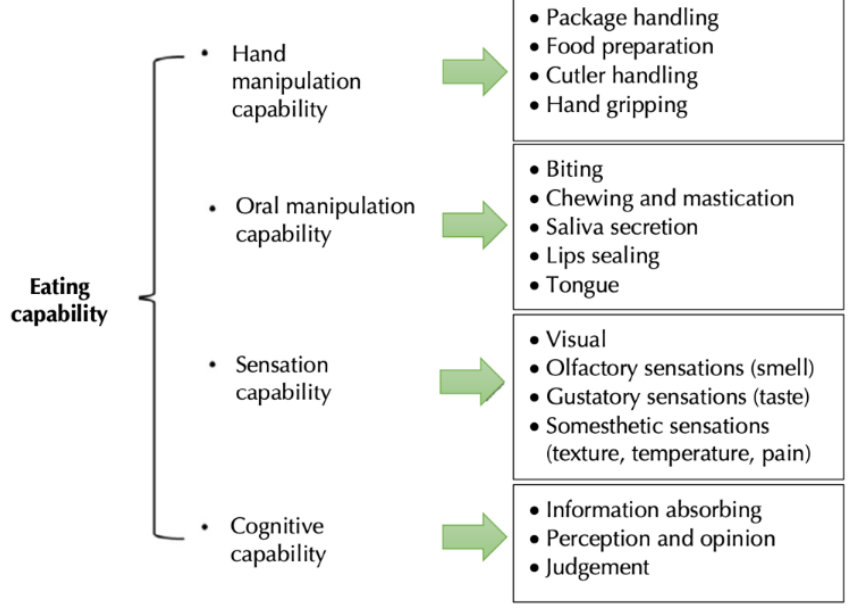

Figure 3. Components of eating capability 16

\section{Hand manipulation capability}

Capability of hand manipulation is required in preparing food and using cutlery. To be able to eat by their selves, people use their hands to open and hold food packages, manage food on the plate, hold and lift objects, and put food into the mouth. Older adults are having difficulties in doing those actions. 18

Impaired hand mobility in older adults is frequently caused by Parkinson's disease and rheumatoid arthritis. Tremor of the hands will trouble older adults to manipulate food, using cutlery, lifting and putting food into the mouth. Muscle weakness also causes problems in accuracy and strength of hand grip. 15

Bohannon et al. in 2006 compared the strength of hand grip between younger and older groups using JAMAR dynamometer. The right 
hand grip strength of men and women in group aged more than 65 years were $28-41.7 \mathrm{~kg}$ and $18-24.2 \mathrm{~kg}$ respectively, while the right hand grip strength of men and women in group aged 20 - 40 years were $53.3-54.1 \mathrm{~kg}$ and $30.6-33.2 \mathrm{~kg}$ respectively. ${ }^{19}$ Laguna et al. in 2015 also found decreased grip strength in older adults, particularly who aged more than 90 years. 20

\section{Oral manipulating capability}

Oral cavity is the first part of gastrointestinal tract. Oral manipulating capability is the ability to change food into swallowable bolus and transfer it safely from oral cavity into the stomach. Oral capability is divided into mastication capability and swallowing capability. Laguna et al. in 2017 described oral manipulating capability as shown in figure 4.18

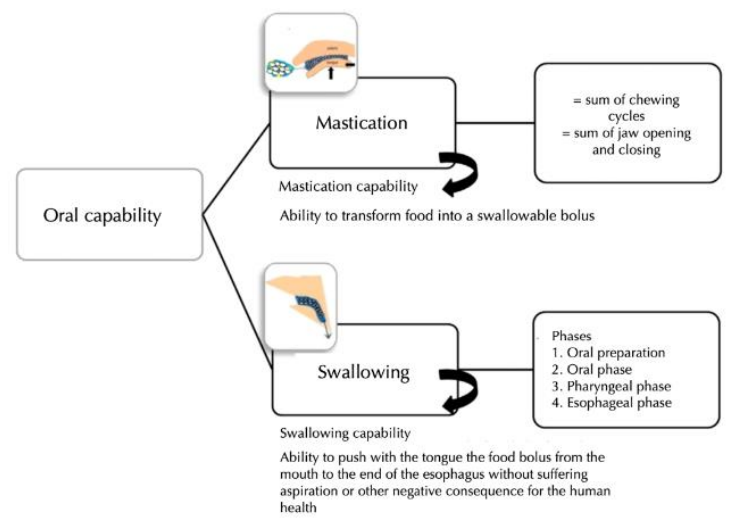

Figure 4. Oral manipulating capability 18

Mastication process is closely related to sensory-motor system involving teeth, orofacial muscles, jaw muscles, lip muscles, tongue, and saliva production. All of those components work in coordinated manner and are controlled by central nervous system to create an efficient mastication process. Any disorder in one or more of those components will impair mastication process. Common disorders in older adults such as loss of dentition, inadequate saliva secretion, tongue problem, and loss of muscle mass that decrease mastication force. ${ }^{18}$

Oral health status especially loss of dentition has significant impact on eating capability of older adults. Several oral diseases such as caries and periodontal illness will cause difficulty in eating, particularly in mastication process. ${ }^{21}$ Bite force decreased significantly in older adults who wore dentures compared to them whose natural teeth were still preserved. The more number of natural teeth, the bite force will get stronger and mastication process will be easier. ${ }^{22}$

Tongue has a very critical role in forming and transferring food bolus.23 Older adults with incomplete dentition swallow a relatively bigger particle even after they try to compensate by chewing longer. In this circumstance, tongue helps to maintain and hold dentures in mouth. Tongue has to position the food while holding dentures. 18 The presence of sarcopenia, which associated to the decreased of tongue strength, will increase the risk of aspiration since the risk of bolus retention in pharynx is also increased. 24 The weakness of the tongue will cause incomplete food clearance, increased time required for eating, decreased food intake, and swallowing difficulty. 18 
Swallowing process consists of three phases in transferring food: oral phase; pharynx phase; and esophageal phase. In older adults, swallowing process is longer, weaker, and less coordinated compared to young adults. Transit from oral phase to pharynx phase in swallowing process also affected by aging. This transit time was reported longer in older adults. ${ }^{25}$ Park et al. in 2017 reported that older adults were having difficulty in swallowing, which measured by increased duration of eating and chewing, also large amount of remnants found in oral cavity and pharynx. 26

\section{Sensation capability}

Sensation capability is the ability to perceive sensory stimuli in the process of eating. Those stimuli are received through five human senses: sight, smell, taste, touch, and hearing. Appearance, flavor, consistency, texture, are all needed in the process of eating for people to enjoy their food.16 Aging causes sensory changes in smell and taste which observed by decreased sensitivity and increased threshold of smell and taste detection. Application of dentures covers the palate and further reduce taste sensation. 25 The decreased sensory perception will reduce the appetite and overall food intake.

\section{Cognitive capability}

Cognitive capability is the ability of an individual to make a decision related to eating, also motoric capability to coordinate all of the actions in eating process. To be able to perform those actions, a good mental capability is required, as well as the ability to receive and process the information such as what is eaten, how frequent, the amount of food eaten, and forth. 16

Patients who have suffered stroke will have difficulty in coordinating actions in eating process, such as inadequate lip closure, mastication, dysphagia and imbalance while sitting. Patients with cognitive disorder like dementia have impairment of sensory perception and food intake control. Eating disorder is also a result from cognitive function disorder. 18

\section{Conclusion}

Decreased nutrition intake in older adults put them in a high risk of malnutrition, especially deficiency of various nutrients. Malnutrition in older adults can be very noxious because it is often recognized too late and its recovery is much more complicated than in younger adults. Moreover, malnutrition in older adults will significantly reduce quality of life, cause deterioration of illness, reduce functional status, increase hospitalization and mortality rate. Hence, optimalization of nutrition intake in older adults is very critical both in healthy and sick older adults. Age-related physiological changes of gastrointestinal tract, social status, economic status, diseases and polypharmacy, and also cognitive problems lead to anorexia and eating difficulty in older adults. Disruption of hand manipulation capability, oral manipulation capability, sensation capability, and cognitive capability will interfere eating capability of older adults. Those problems need to be recognized early so that clinicians and family members can help them obtain adequate nutrition intake and prevent malnutrition.

\section{References}

1. Bulut EA, Khoury R, Lee H, Grossberg GT. Eating disturbances in the elderly: A geriatric-psychiatric perspective. Nutr Heal Aging. 2019; 5(3): 185-98.

2. Setiati $\mathrm{S}$, Istanti $\mathrm{R}$, Andayani $\mathrm{R}$, Kuswardhani RAT, Aryana IGPS, Putu ID, 
et al. Cut-off of anthropometry measurement and nutritional status among elderly outpatient in Indonesia: multi-centre study. Acta Med Indones. 2010; 42(4): 224-30.

3. Corcoran C, Murphy C, Culligan EP, Walton J, Sleator RD. Malnutrition in the elderly. Sci Prog. 2019; 102(2): 171-80.

4. Wallace JI. Malnutrition and enteral/parenteral alimentation. In: Halter JB, Ouslander JG, Studenski S, High KP, Asthana S, Ritchie CS et al, editor. Hazzard's Geriatric Medicine and Gerontology. 7th ed. New York: McGraw Hill Education; 2017; 511-24.

5. Donini LM, Poggiogalle E, Balzo V. Food preferences in the elderly: molecular basis. Mol Basis Nutr Aging [Internet]. 2016; 121-6. Available from: http://dx.doi.org/10.1016/B978-0-12801816-3.00009-1

6. Volkert D, Beck AM, Cederholm T, CruzJentoft A, Goisser S, Hooper L, et al. ESPEN guideline on clinical nutrition and hydration in geriatrics. Clin Nutr [Internet]. 2019; 38(1): 10-47. Available from:

https://doi.org/10.1016/j.clnu.2018.05. 024

7. Wendt J, Rotkiewicz A, Berg A. Geriatric nutrition. In: Kogan M, editor. Integrative Geriatric Medicine. Canada: Oxford University Press; 2018; 10-26.

8. Johnson CS, Sacks G. Nutrition and aging. In: Fillit HM, Rockwood K YJ, editor. Brocklehurst's Textbook of Geriatric Medicine and Gerontology. 8th ed. Philadelphia: Elsevier; 2017; 660-6.

9. Hall KE. Aging of the gastrointeestinal system. In: Halter JB, Ouslander JG, Studenski S, High KP, Asthana S, Ritchie
CS et al, editor. Hazzard's Geriatric Medicine and Gerontology. 7th ed. New York: McGraw Hill Education; 2017; $1333-40$.

10. Calabrese JM, Jones JA. Oral health. In: Halter JB, Ouslander JG, Studenski S, High KP, Asthana S, Ritchie CS et al, editor. Hazzard's Geriatric Medicine and Gerontology. 7th ed. New York: McGraw Hill Education; 2017; 546-58.

11. Di Francesco V, Pellizzari L, Corrà L, Fontana G. The anorexia of aging: impact on health and quality of life. Geriatr Care. 2018; 4(2): 21-8.

12. Abu-Ghanem S, Chen S, Amin MR. Oropharyngeal dysphagia in the elderly: evaluation and prevalence. Curr Otorhinolaryngol Rep. 2020;8(1):34-42.

13. Berry EM, Marcus EL. Disorders of eating in the elderly. J Adult Dev. 2000; 7(2): 8799.

14. Bender DV, Krznarić Ž. Nutritional issues and considerations in the elderly: an update. 2020; 180-3.

15. Nyberg M, Olsson V, Pajalic Z, Örtman G, Andersson HS, Blucher A, et al. Eating difficulties, nutrition, meal preferences and experiences among elderly a literature overview from a scandinavian context. J Food Res. 2014; 4(1): 22.

16. Laguna L, Chen J. The eating capability: constituents and assessments. Food Qual Prefer. 2016; 48: 345-58.

17. Westergren A, Unosson M, Ohlsson O, Lorefält B, Hallberg IR. Eating difficulties, assisted eating and nutritional status in elderly ( $\geq 65$ years) patients in hospital rehabilitation. Int $\mathrm{J}$ Nurs Stud. 2002; 39(3): 341-51.

18. Laguna L, Sarkar A, Chen J. eating capability assessments in elderly 
populations [Internet]. Nutrition and Functional Foods for Healthy Aging. Elsevier Inc.; 2017. 83-98 p [cited 14 December 2020]. Available from: http:/ /dx.doi.org/10.1016/B978-0-12805376-8.00010-1

19. Bohannon RW, Peolsson A, MassyWestropp N, Desrosiers J, Bear-Lehman J. Reference values for adult grip strength measured with a Jamar dynamometer: a descriptive meta-analysis. Physiotherapy. 2006; 92(1): 11-5.

20. Laguna L, Sarkar A, Artigas G, Chen J. A quantitative assessment of the eating capability in the elderly individuals. Physiol Behav [Internet]. 2015;147:27481 [cited 13 December 2020]. Available from:

http://dx.doi.org/10.1016/j.physbeh.20 15.04 .052

21. Antoniadou M, Varzakas T. Breaking the vicious circle of diet, malnutrition and oral health for the independent elderly. Crit Rev Food Sci Nutr. 2020; 1-23.

22. Sarkar A. Oral processing in elderly: understanding eating capability to drive future food texture modifications. Proceedings of the Nutrition Society. 2019; 78(3): 329-39.

23. Arakawa I, Igarashi K, Imamura Y, Müller F, Abou-Ayash S, Schimmel M. Variability in tongue pressure among elderly and young healthy cohorts: A systematic review and meta-analysis. J Oral Rehabil. 2020;(July): 1-19.

24. Cichero JAY. Age-related changes to eating and swallowing impact frailty: Aspiration, choking risk, modified food texture and autonomy of choice. Geriatr. 2018; 3(4): 1-10.

25. Britton D. the impact of aging and progressive neurological disease on swallowing: a concise overview. J Texture Stud. 2016; 47(4): 257-65.

26. Park HS, Kim D, Lee SY PK. The effect of againg on mastication and swallowing parameters according to the hardness change of solid food. 2017;1-29 [cited 15 December 2020]. Available from: http://dx.doi.org/10.1111/jtxs. 12249. 